\title{
Bleeding with gastrointestinal and ureteral ulcers after gefitinib treatment: a case report
}

\author{
Jian Xiao ${ }^{1} \wedge$, Wenjing Ruan ${ }^{1,2}, \mathrm{Ning} \mathrm{Li}^{1}$, Dongdong Huang ${ }^{1}$ \\ ${ }^{1}$ Department of Respiratory and Critical Care Medicine, the Fourth Affiliated Hospital Zhejiang University School of Medicine, Yiwu, China; \\ ${ }^{2}$ Department of Respiratory Disease, Sir Run Run Shaw Hospital, Zhejiang University School of Medicine, Hangzhou, China \\ Correspondence to: Wenjing Ruan, MD, PhD. Department of Respiratory and Critical Care Medicine, the Fourth Affiliated Hospital Zhejiang \\ University School of Medicine, Yiwu, China; Department of Respiratory Disease, Sir Run Run Shaw Hospital, Zhejiang University School of \\ Medicine, Hangzhou, China. Email: rwj@zju.edu.cn.
}

\begin{abstract}
Epidermal growth factor receptor (EGFR) inhibitors, as a first-line drug treatment in the EGFRsensitive mutation of advanced non-small-cell lung cancer (NSCLC), has been used in a wide variety of malignancies. These therapies have various troublesome side effects including diarrhea, stomatitis, mucositis, rash, dry skin and paronychia which may impact a patient's clinical outcome in addition to their beneficial effects. Here, we report a rare case of a 69-year-old male having advanced NCSLC treated with gefitinib, who developed EGFR tyrosine kinase inhibitor (TKI)-related multiple ulcers accompanied by bleeding. After a detailed examination and multidisciplinary discussion, we have learnt that early identification of gastrointestinal (GI) bleeding and blood in urine is due to targeted drugs rather than other causes such as ulcer and stones. Good results have also been achieved by reducing drug dosage under close observation. So far, the patient has been followed up for 15 months, and his condition remained stable. Up to now, there is no case of such severe side effect having been found, and no guidelines recommended for handling such adverse events. Through clinical case sharing, early recognition and proactive management are particularly important in order to minimize appropriately the effect of these adverse events. The whole course of a disease can be vividly illustrated through a case report, so as to provide more effective guiding principles for clinicians.
\end{abstract}

Keywords: Gefitinib; bleeding with ulcers; side effect; non-small cell lung cancer (NSCLC); case report

Submitted Dec 10, 2020. Accepted for publication Mar 05, 2021.

doi: $10.21037 /$ tcr-20-3420

View this article at: http://dx.doi.org/10.21037/tcr-20-3420

\section{Introduction}

As is well-known, lung cancer has been the leading cause of cancer mortality globally. Non-small cell lung cancer (NSCLC) mainly consists of adenocarcinoma and squamous cell carcinoma. At present, target therapy drugs are widely used, and increasingly, rare adverse reactions are emerging, although recommended guidelines are not updated in time (1-3). This requires clinical case sharing to obtain clinical evidence for reasonable and effective intervention $(4,5)$.
Here, we present a rare case of first-generation targeted drugs developing epidermal growth factor receptor tyrosine kinase inhibitor-related (EGFR-TKI-related) multiple ulcers accompanied by bleeding. In particular, discussion of the case and treatment based on multidisciplinary staff involvement are emphasized in this case. The following case is presented in accordance with the CARE reporting checklist (available at http://dx.doi.org/10.21037/tcr-203420).

^ ORCID: 0000-0001-9863-8704. 

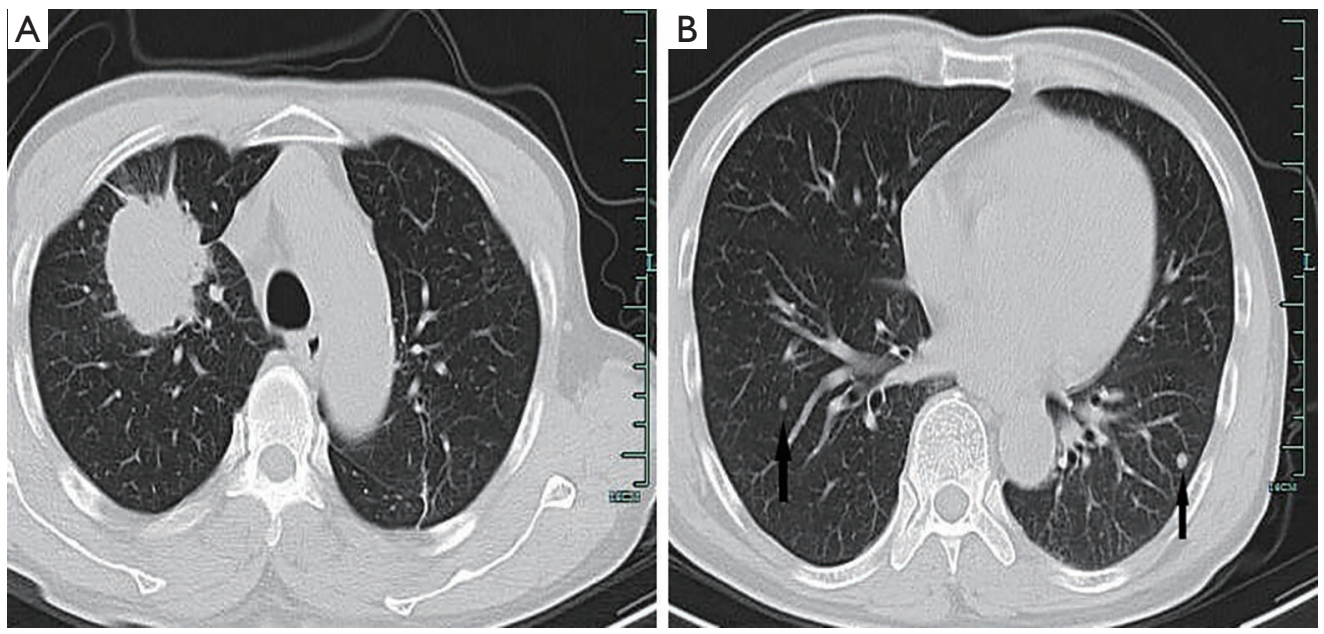

Figure 1 (A,B) CT of the chest in January 2019. (B, the black arrows indicate intrapulmonary metastasis of lung cancer).
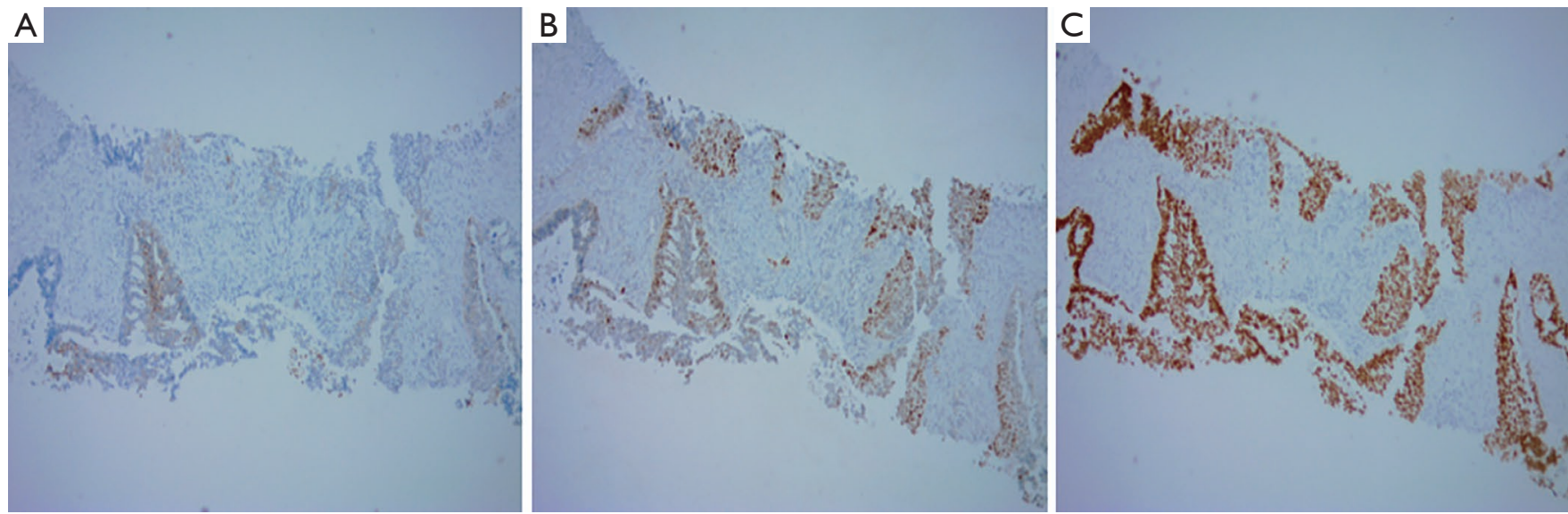

Figure 2 (A,B,C) Pathological images of lung biopsy with immunohistochemical EnVison method (A, Napsin A, original ×50. B, P63, original $\times 50$. C, TTF-1, original $\times 40)$.

\section{Case presentation}

In January 2019, a 69-year-old man presented with dry cough for two months and hemoptysis for one week. Computed tomography (CT) of the chest revealed a right upper lung mass with metastatic lesions throughout the lungs and mediastinal lymph nodes (Figure 1A,B). A CT-guided biopsy from the right lung mass was suggestive of adenocarcinoma on the basis of positive immunohistochemistry (IHC) for Napsin A, P63 and thyroid transcription factor 1 (Figure $2 A, B, C$ ). According to the International Union Against Cancer (Eighth Edition), the TNM classification was T3N3M1a. The patient had an exon 19 deletion (EGFR-p.Leu747_Pro753delinsSer). In view of stage IV lung adenocarcinoma and the EGFR mutation being positive, gefitinib (Qilu Pharmaceutical Co., Ltd., Hainan China, $250 \mathrm{mg}$ po, once a day), was given as the first-line systemic therapy, starting in February 2019. After two months, the patient showed a good clinical response to treatment to judge by a reduction in the size of the lumps (Figure 3A,B).

After five months of gefitinib treatment, the patient was admitted to the department of urology with abdomen discomfort and hematuria. On admission, urinalysis showed that the red blood cell count was $8,594.7 / \mu \mathrm{L}$, the other cell types were normal, erythrocyte morphology was of 

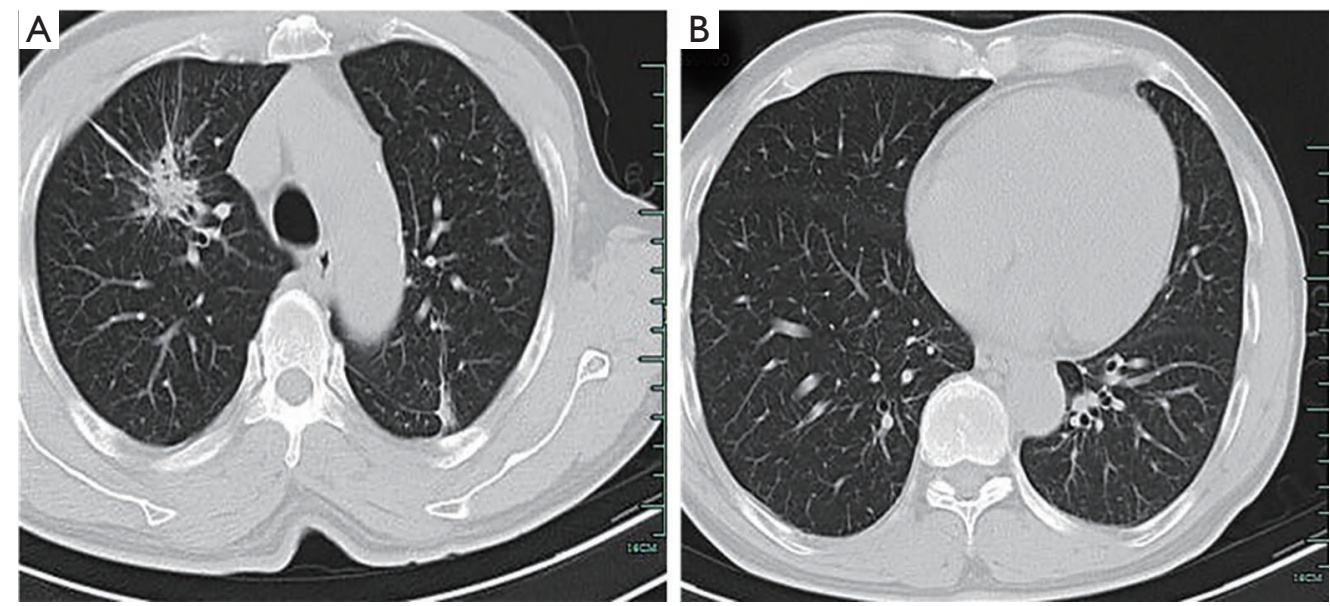

Figure 3 (A,B) CT image of chest two months after initiation of gefitinib.

one type, and the white blood cell count was normal. The results of urinary cytology were negative for malignant cell. The ultrasonogram indicated kidney stone. Follow-up radiographs showed a considerable decrease in size of the mass in the right upper lobe. On ureteroscopy, the left upper ureter was found to be surrounded by mucous membrane ulcer near the mouth of the renal pelvis (Figure $4 A$ ), accompanied by hemorrhage, and a $0.8 \mathrm{~cm}$ stone was located in the lower calyces of the right kidney. The patient was treated with holmium laser lithotripsy through a ureteroscope, and ureteral stents inserted bilaterally. The operation proceeded unproblematically and the postoperative recovery was good. Histopathological inspection of the ureteral ulcer revealed no tumor cells. Three weeks post-operatively, the ureteral stents were removed, but hematuria was still present. Repeat urinalysis showed that the red blood cell count was more than $100 / \mu \mathrm{L}$. On account of bleeding from the multiple ulcers, the patient's hemoglobin was low, and the value fluctuated between 90 and $100 \mathrm{~g} / \mathrm{dL}$. Clotting function and platelets were stable during hospitalization. The patient had been taking no drugs that might simultaneously enhance the blood concentration of gefitinib or to affect the blood system. In addition, he took no medication that would induce ulcer, such as nonsteroidal anti-inflammatory drugs (NSAIDs) or antiplatelet drugs. Taking into account the side effects of gefitinib, the absence of gastrointestinal (GI) and urinary tract disease from the patient's history, the related literature and multidisciplinary expertise, it was concluded that the ulcers were caused by gefitinib administration. In combination with patient tolerability and the need for comprehensive treatment, surgeons and physicians sought the patient's consent to continue to use this agent.

After forty days, the patient was readmitted to hospital because of hematemesis, acid reflux and skin rash. Emergency gastroscopy confirmed multiple ulcers of the oral cavity, esophagus and stomach accompanied by bleeding (Figure 4B,C,D). Histopathology of the digestive tract ulcer revealed no tumor cells. CT of the chest showed stable disease (SD). Urinalysis revealed a red blood cell count of $42,172.5 / \mu \mathrm{L}$, the remaining cell types were normal. After informing the patient of the side effects of gefitinib, he agreed to cease having this agent. The patient gradually recovered from the illness after fasting, stomach protection, hemostasis and skin treatment. After two weeks, re-examination by gastroscopy (Figure $4 E, F$ ) and urinalysis showed that hematemesis and hematuria were improved, and gastroscopic pathology and urinary cytology was negative for malignant cells. Oral gefitinib was re-started at half the previous dose (125 mg once daily) for treatment of the lung cancer. With this decreased oral medication, the digestive and urinary symptoms re-appeared, but the degree was lighter than before. Until April 2020, the patient was found to have cerebral metastasis indicating tumor progression. The targeted drug was replaced by osimertinib (AstraZeneca Pharmaceutical Co. Ltd., UK, $80 \mathrm{mg}$ po, once a day) after testing for T790 mutations. All procedures performed in studies involving this patient were in accordance with the ethical standards of the institutional and/or national research committee(s) and with the Helsinki Declaration (as revised in 2013). Written informed consent was obtained from the patient. 

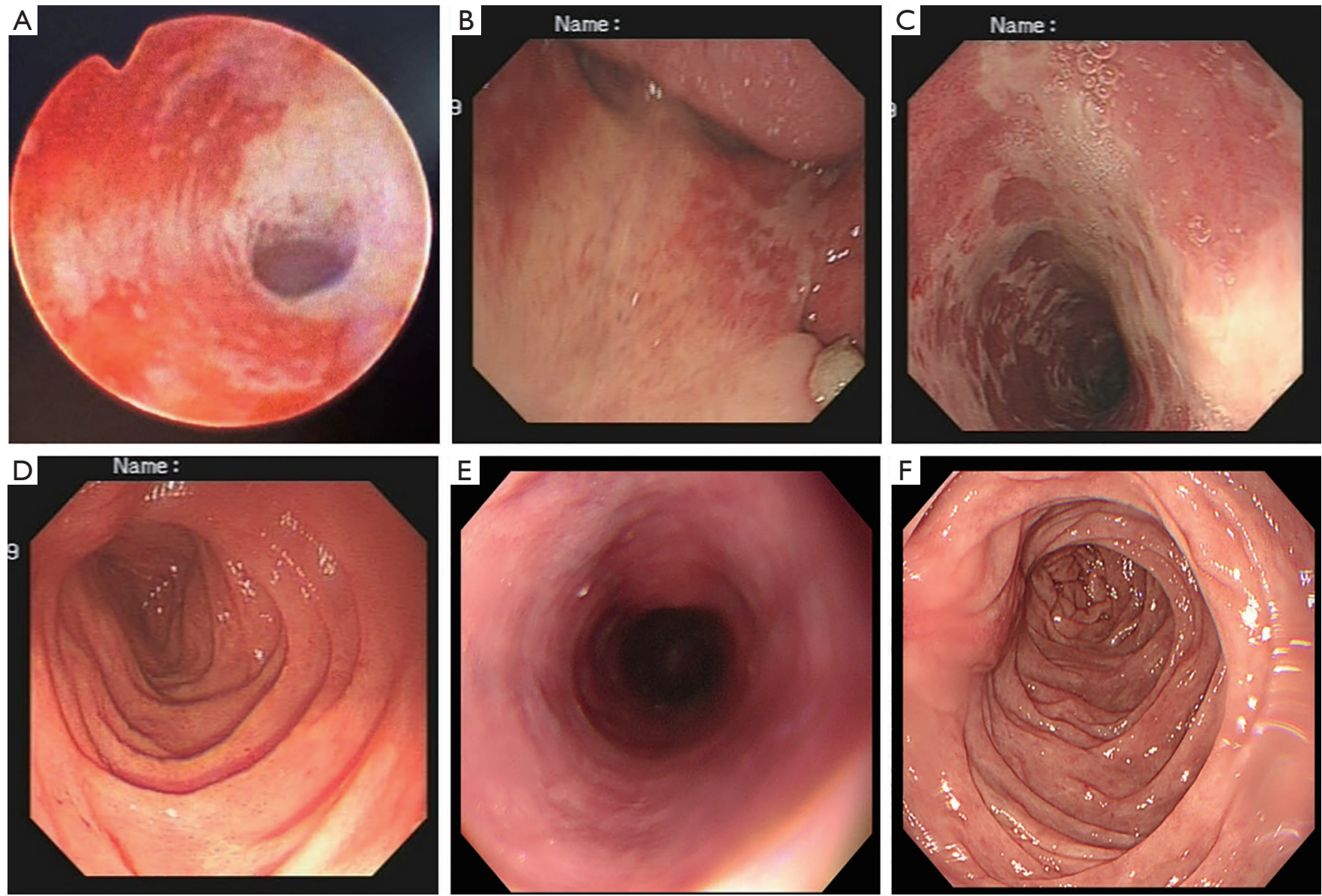

Figure 4 (A) The ureteral ulcer. (B,C,D) Emergency gastroscopy (B, oral mucosal ulcer. C, esophageal ulcers. D, descending duodenal ulcer). (E,F) Reexamination of gastroscopy (E, an improved esophageal ulcer after drug withdrawal. (F) A descending duodenal ulcer that has improved).

\section{Discussion}

Multi-system ulcer with bleeding is not a common manifestation, its incidence having been reported as being up to $8.7 \%$ (6-8). The molecularly targeted drugs cause side effects reported mainly as skin reaction, liver function damage and oral cavity mucositis, but multi-system ulcer is rare. The adverse effect mentioned in this case has a complex mechanism. Clinicians need to identify the reasons in order to understand subsequent intervention. Whether the toxicities are correlated with different varieties or mutation types remains to be studied. Bleeding with gastrointestinal and ureteral ulcers, as grade $\geq 3$ toxicity, may also be caused by chemical compounds, irradiation, infection, and metastasis, such that early differential diagnosis is especially important. Once multiple ulcers with bleeding occurs, it easily causes serious complication such as GI or ureter perforation (9). Our patient was a previously healthy male without history of mucosal or ureteral ulcer. At the same time, reviewing the patient's history, we found that the patient was not taking drugs such as statins that increase the required concentration of gefitinib. Combining this information with the pathologic findings suggested that multiple ulcers were not caused by tumor metastasis and factors in the patient history, and it seems that EGFR-TKIrelated side effects induced oral, esophageal, gastric and ureteral ulcers. It is worth noting that pathologic findings are need for the differential diagnosis between metastasis and drug-induced ulcers; the former shows ulceration due to tumor necrosis, while the latter lacks evidence of tumor (10). So far, there are no formal official global guidelines for the 
management of adverse events from EGFR tyrosine kinase inhibitors. Therefore, we had to take the decision to replace this with another drug or reduce the dosage of the initial agent according to regional expert consensus or published case reports. The former choice was not invalid, however, the decreased dosage option proved to be effective, which also provided theoretical support for clinical treatment.

NSCLC represents $85 \%$ of all lung cancer diagnoses and is a heterogeneous disease with several biological events driving tumor growth and progression (11). AntiEGFR therapy forms the backbone of treatment in lung cancer with sensitive EGFR mutations. Especially for Asian patients with NSCLC who have up to $50 \%$ drug-sensitive EGFR mutations such as the exon 19 deletion like our patient, they can select the agents as first-line therapies (12). Gefitinib shows a rapid and dramatic response in treating the select population (13). The mechanism for developing multiple ulcers with bleeding is still unknown. Gefitinib is known to have antitumor vascular effects as a tyrosine kinase inhibitor. Whether rapid regression of the tumor increases the mucosal toxicity of EGFR-TKIs to make it susceptible to additive damage such as multiple ulcers is an idea that will need more research.

While common toxicities such as stomatitis, mucositis and skin rash following treatment are well documented responses $(14,15)$, bleeding with multiple ulcers has not been reported. Clearly, internal mucosal ulcers are not as easily diagnosed as in the skin. We think it is important to report our experience for clinicians to update their understanding of GI bleeding occurring in the setting of primary ulcer and secondary targeted drugs.

\section{Acknowledgments}

We would like to thank Dr. Brian Eyden (Manchester, United Kingdom) for final English-language assistance with the manuscript.

Funding: None.

\section{Footnote}

Reporting Checklist: The authors have completed the CARE reporting checklist. Available at http://dx.doi.org/10.21037/ tcr-20-3420

Conflicts of Interest: All authors have completed the ICMJE uniform disclosure form (available at http://dx.doi. org/10.21037/tcr-20-3420). The authors have no conflicts of interest to declare.

Ethical Statement: The authors are accountable for all aspects of the work in ensuring that questions related to the accuracy or integrity of any part of the work are appropriately investigated and resolved. All procedures performed in studies involving human participants were in accordance with the ethical standards of the institutional and/or national research committee(s) and with the Helsinki Declaration (as revised in 2013). Written informed consent was obtained from the patient.

Open Access Statement: This is an Open Access article distributed in accordance with the Creative Commons Attribution-NonCommercial-NoDerivs 4.0 International License (CC BY-NC-ND 4.0), which permits the noncommercial replication and distribution of the article with the strict proviso that no changes or edits are made and the original work is properly cited (including links to both the formal publication through the relevant DOI and the license). See: https://creativecommons.org/licenses/by-nc-nd/4.0/.

\section{References}

1. Kris MG, Natale RB, Herbst RS, et al. Efficacy of gefitinib, an inhibitor of the epidermal growth factor receptor tyrosine kinase, in symptomatic patients with non-small cell lung cancer: a randomized trial. JAMA 2003;290:2149-58.

2. Ricciardi S, Tomao S, de Marinis F. Toxicity of targeted therapy in non-small-cell lung cancer management. Clin Lung Cancer 2009;10:28-35.

3. Califano R, Tariq N, Compton S, et al. Expert Consensus on the Management of Adverse Events from EGFR Tyrosine Kinase Inhibitors in the UK. Drugs 2015;75:1335-48.

4. Luo C, Lv M, Li Y, et al. Gefitinib-induced interstitial pneumonia: A case report and review of the literature. Exp Ther Med 2014;7:855-9.

5. Dai J, Belum VR, Wu S, et al. Pigmentary changes in patients treated with targeted anticancer agents: A systematic review and meta-analysis. J Am Acad Dermatol 2017;77:902-10.e2.

6. Inoue A, Kobayashi K, Maemondo M, et al. Updated overall survival results from a randomized phase III trial comparing gefitinib with carboplatin-paclitaxel for chemo- 
naive non-small cell lung cancer with sensitive EGFR gene mutations (NEJ002). Ann Oncol 2013;24:54-9.

7. $\mathrm{Wu} \mathrm{YL}, \mathrm{Zhou} \mathrm{C}, \mathrm{Hu} \mathrm{CP}$, et al. Afatinib versus cisplatin plus gemcitabine for first-line treatment of Asian patients with advanced non-small-cell lung cancer harbouring EGFR mutations (LUX-Lung 6): an open-label, randomised phase 3 trial. Lancet Oncol 2014;15:213-22.

8. Sanford M, Scott LJ. Gefitinib: a review of its use in the treatment of locally advanced/metastatic non-small cell lung cancer. Drugs 2009;69:2303-28.

9. Kim MS, Kook EH, Ahn SH, et al. Gastrointestinal metastasis of lung cancer with special emphasis on a longterm survivor after operation. J Cancer Res Clin Oncol 2009;135:297-301.

10. Chung WP, Song HL, Ho CL, et al. Gastric perforation secondary to regression of lung adenocarcinoma after gefitinib treatment. J Clin Oncol 2013;31:e6-8.

Cite this article as: Xiao J, Ruan W, Li N, Huang D. Bleeding with gastrointestinal and ureteral ulcers after gefitinib treatment: a case report. Transl Cancer Res 2021;10(4):1941-1946. doi: $10.21037 /$ tcr-20-3420
11. Peters S, Adjei AA, Gridelli C, et al. Metastatic nonsmall-cell lung cancer (NSCLC): ESMO Clinical Practice Guidelines for diagnosis, treatment and follow-up. Ann Oncol 2012;23 Suppl 7:vii56-64.

12. Hirsch FR, Bunn PA Jr. EGFR testing in lung cancer is ready for prime time. Lancet Oncol 2009;10:432-3.

13. Liu Z, Liu L, Li M, et al. Epidermal growth factor receptor mutation in gastric cancer. Pathology 2011;43:234-8.

14. Park K, Tan EH, O'Byrne K, et al. Afatinib versus gefitinib as first-line treatment of patients with EGFR mutationpositive non-small-cell lung cancer (LUX-Lung 7): a phase 2B, open-label, randomised controlled trial. Lancet Oncol 2016;17:577-89.

15. Cubero DIG, Abdalla BMZ, Schoueri J, et al. Cutaneous side effects of molecularly targeted therapies for the treatment of solid tumors. Drugs Context 2018;7:212516. 